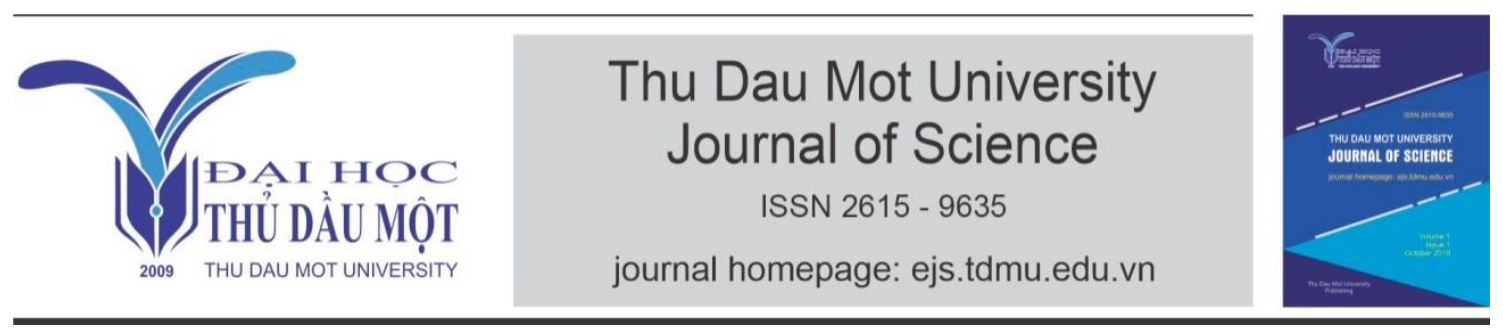

\title{
Diversity of Orchidaceae in South East Viet Nam
}

by Nguyen Minh Ty, Nguyen Vinh Hien (Thu Dau Mot University)

\author{
Article Info: Received 4 Feb. 2021, Accepted 1 Mar. 2021, Available online 15 Mar. 2021 \\ Corresponding author: tynm@tdmu.edu.vn, hiennv@tdmu.edu.vn \\ https://doi.org/10.37550/tdmu.EJS/2021.01.150
}

\begin{abstract}
Result of study on the diversity of orchids in Southern East Viet Nam at 6 study sites from July 2019 to October 2020 have identified 173 orchids species belong to 50 diffrent genus, accounting for $14.41 \%$ of the total number of orchid species available in Viet Nam (1.200 species). Among them, all species were used as ornamental plants; 13 species were used for medicinal herbs; and 6 species were listed for conservation in Vietnam Red Data Book, (2019). Fresh samples are processed and photographed in the field, then have brought back to plant and take care of the garden of Thu Dau Mot University. The life forms of orchids were divided into groups including: (1) epiphytes with 144 species, (2) terrestrials with 26 species, (3) saprophytes with 1 species, (4) lithophytes with 2 species. Added 60 species to forest orchid flora of Southern East Viet Nam.
\end{abstract}

Keywords: Diversity of Orchidaceae family, Southern East Viet Nam

\section{Introduction}

The South East is located in wide plains transitioning from the South Central Highlands to the Mekong river delta, including 6 provinces (Ho Chi Minh City, Ba Ria-Vung Tau, Tay Ninh, Binh Duong, Binh Phuoc, Dong Nai). The elevation of the different topographic from 200 to $750 \mathrm{~m}$. The highest is Ba Den mountain with $986 \mathrm{~m}$ in Tay Ninh province and is characterized by a sub-equatorial climate with high temperature and almost unchanged throughout the year. The typical habitats in South East region are evergreen forest, deciduous forest, mangrove forest and grassland with a very diverse flora, especially forest orchids (Sterling, Hurley \& Minh, 2007). Studies of orchids in South East have not been much and full, mainly focused in national parks, nature reserves and special forest (Cat Tien national park and Vinh Cuu nature reserve in Dong 
Nai province, Bu Gia Map national park in Binh Phuoc province, Lo Go-Xa Mat national park in Tay Ninh province, Binh Chau Phuoc Buu nature reserve in Ba Ria Vung Tau province). The South East is considered as a conservation area for rare and economically valuable orchid species in South of Viet Nam. Over the past decades, due to many human impacts such as: forest exploitation, cultivation, industrial tree planting, forestry, construction of irrigation, hydropower and urbanization etc...Its has narrowed the natural forest area, habitats have been changed or lost, many species of forest plants are threatened in which, species of forest orchids are reducing rapidly. Especially species of economic value, rare medicinal. Therefore, the investigation and assessment of the reality forest orchid species composition as a basis for the management, conserve precious genetic resources and maintain plant biodiversity is necessary.

\section{Material and research methods}

\section{Materials}

All species of the orchid family (Orchidaceae) are distributed in South East region Viet Nam, organic fertilizer, coir, charcoal for use in orchid cultivation.

\section{Research methods}

Surveying and sampling of orchid species (Orchidaceae) in the field, the survey location was established based on the distribution characteristics of the orchid family (Orchidaceae), in which priority for investigation in national parks, nature reserves and primeval forests at 6 study sites from July 2019 to October 2020: Cat Tien national park and Vinh Cuu nature reserve in Dong Nai province, Bu Gia Map national park in Binh Phuoc province, Lo Go-Xa Mat national park in Tay Ninh province, Binh Chau Phuoc Buu nature reserve in Ba Ria Vung Tau province and Dau Tieng Binh Duong province.

In each study site is divided according to the survey route and sample collection 2 times a year in the rainy and dry seasons.

Directly collect samples with people specializing in exploiting forest orchids at 6 study sites.

Orchid samples collected during the survey included fresh samples, processed and photographed in the field, and accompanied by sample records. The fresh samples are kept at the orchid collection garden of Thu Dau Mot University.

Scientific names and life forms of species of the orchid family (Orchidaceae) were determined according to the comparative morphological method on the basis of published documents of Pham Hoang Ho (2003, 2006), Gagnepain \& Guillaumin (1932- 1934), Averyanov et al., (2003), Cribb (1998), Averyanov et al. (2003), Averyanov et al., (2013) at the same time comparing with the standard sample stored in 
the Botanical Museum of the Institute of Tropical Biology. The editing and updating of the scientific names of the species in the (Orchidaceae) is conducted according to the publications of Kew science (https://wcsp.science.kew.org) and The Plan List (http://www .theplantlist.org/).

\section{Results and Discussion}

\subsection{Species composition diversity}

The results of analyzing data obtained in the field and in the laboratory have identified the orchid family (Orchidaceae) in the South East region with 173 species belonging to 50 genera, of which 60 species were added to the South East compared with previous studies (Table 1).

TABLE 1. List of species composition of the orchid family (Orchidaceae) in South East region Viet Nam

\begin{tabular}{|c|c|c|c|}
\hline STT & Science name & Vietnamese name & $\begin{array}{l}\text { Life } \\
\text { form }\end{array}$ \\
\hline 1 & Acampe carinata (Griff.) Panigrahi & A cam sóng & Epi \\
\hline 2 & Acampe ochracea (Lindl.) Hochr. & A cam sét & Epi \\
\hline 3 & Acampe papillosa Lindl. $^{*}$ & Bắp ngô cụm ngắn & Epi \\
\hline 4 & Acriopsis indica C.Wright & Tổ yến ấn & Epi \\
\hline 5 & Acriopsis liliifolia (J.Koenig) Ormerod & Tố yến Java & Epi \\
\hline 6 & Aerides falcata Lindl. \& Paxton & Giáng hương & Epi \\
\hline 7 & Aerides houlletiana Rchb.f. & Giáng hương quế nâu & Epi \\
\hline 8 & Aerides multiflora Roxb." & Giáng hương đuôi cáo & Epi \\
\hline 9 & Aerides odorata Lour. & Lan giáng hương & Epi \\
\hline 10 & Aerides rosea Lodd. ex Lindl. \& Paxton & Giáng xuân nhiều hoa & Epi \\
\hline 11 & Agrostophyllum callosum Rchb.f. & Càng cua thân dài & Epi \\
\hline 12 & Agrostophyllum planicaule (Wall. ex Lindl.) Rchb.f. & Xích hủ thân mập & Epi \\
\hline 13 & Anoectochilus roxburghii. ${ }^{*}$ & Lan Kim tuyến & Ter \\
\hline 14 & Aphyllorchis montana Rchb.f. & Âm lan núi & Sap \\
\hline 15 & Appendicula cornuta Blume & Vệ lan móng & Lit, Epi \\
\hline 16 & Appendicula floribunda (Schltr.) Schltr. & Vệ lan nhiều hoa & Lit, Epi \\
\hline 17 & Arachnis labrosa (Lindl. \& Paxton) Rchb.f. & Lan nhện môi hẹp & Epi \\
\hline 18 & Biermannia sigaldii Seidenf. & Lan bạch manh & Epi \\
\hline 19 & Brachypeza laotica (Seidenf.) Seidenf. & Lan môi sừng & Epi \\
\hline 20 & Bulbophyllum affine Wall. ex Lindl." & Cầu điệp gối & Epi \\
\hline 21 & Bulbophyllum ayuthayense J.J.Verm., Schuit. \& de Vogel* & Lan củ chén xiêm & Epi \\
\hline
\end{tabular}




\begin{tabular}{|c|c|c|c|}
\hline 22 & Bulbophyllum bariense Gagnep ${ }^{*}$ & Lọng bà rịa & Epi \\
\hline 23 & Bulbophyllum blepharistes Rachb.f. & Lọng tai thỏ & Epi \\
\hline 24 & Bulbophyllum careyanum (Hook. f.) Spreng. & Cầu diệp trên & Epi \\
\hline 25 & Bulbophyllum dissitiflorum Seidenf $^{*}$ & Lọng chùm cong & Epi \\
\hline 26 & Bulbophyllum haniffii Carr & Lọng chân rết & Epi \\
\hline 27 & Bulbophyllum hymenanthum Hook. f. & Cầu diệp màng & Epi \\
\hline 28 & Bulbophyllum macranthum Lindl & Lọng hoa lớn & Epi \\
\hline 29 & Bulbophyllum macrocoleum Seidenf. & Cầu diệp cô lê & Epi \\
\hline 30 & Bulbophyllum morphologorum Kraenzil. & Cầu diệp & Epi \\
\hline 31 & Bulbophyllum refractum (Zoll.\&Mor.) Reichbf. & Cầu điệp thòng & Epi \\
\hline 32 & Bulbophyllum reptans ( Lindl.) Lindl. & Cầu điệp bò & Epi \\
\hline 33 & Bulbophyllum retusiusculum Rchb.f. & Cầu diệp tà & Epi \\
\hline 34 & Bulbophyllum rufinum Rchb.f. & Cầu diệp sói & Epi \\
\hline 35 & Bulbophyllum wallichii Rchb.f. & Cầu diệp wallich & Epi \\
\hline 36 & Calanthe alleizettei Gagnep. & Bầu rượu & Ter \\
\hline 37 & Calanthe angustifolia (Blume) Lindl. ${ }^{*}$ & Bầu rượu kim tân & Ter \\
\hline 38 & Calanthe chevalieri Gagnep & Bầu rượu Chevalier & Ter \\
\hline 39 & Calanthe clavata Lindl." & Bầu rượu chùy & Ter \\
\hline 40 & Calanthe rubens Ridl. ${ }^{*}$ & Bầu rượu xuân & Ter \\
\hline 41 & Calanthe triplicata (Willemet) Ames & Kiều lan xếp ba & Ter \\
\hline 42 & Calanthe vestita Lindl." & Bầu rượu tím & Ter \\
\hline 43 & Cleisostoma arietinum (Rchb.f.) Garay ${ }^{*}$ & Mật khẩu đầu bò & Epi \\
\hline 44 & Cleisostoma birmanicum (Shltr.) Garay. & Mật khẩu Miến Điện & Epi \\
\hline 45 & Cleisostoma duplicilobum (J.J.Sm.) Garay & Mật khẩu hai thùy & Epi \\
\hline 46 & Cleisostoma fuerstenbergianum Kraenzl. $^{*}$ & Mật khẩu mành & Epi \\
\hline 47 & Cleisostoma lecongkietii Tich et Aver ${ }^{*}$ & $\begin{array}{l}\text { Mật khẩu Lê Công } \\
\text { Kiêt }\end{array}$ & Epi \\
\hline 48 & Cleisostoma racemiferum (Lindl.) Garay & Mật khẩu chia nhánh & Epi \\
\hline 49 & Coelogyne assamica Linden \& Rchb.f. ${ }^{*}$ & Thanh đạm cánh bướm & Epi \\
\hline 50 & Coelogyne brachyptera Rchb.f. & Thanh đạm xanh & Epi \\
\hline 51 & Coelogyne fimbriata Lindl. ${ }^{*}$ & Thanh đạm rìa & Epi \\
\hline 52 & Coelogyne flaccida Lindl. & Thanh đạm mềm & Epi \\
\hline 53 & Coelogyne moorena Rolfe $^{*}$ & Thanh đạm tuyết ngọc & Epi \\
\hline 54 & Coelogyne sanderae Kraenzl. ex O'Brien ${ }^{*}$ & Thanh đạm cảnh & Epi \\
\hline 55 & Coelogyne trinervis Lindl. & Thạch đạm ba gân & Epi \\
\hline 56 & Coelogyne viscosa Rchb.f. & Thanh đạm cỏ & Epi \\
\hline 57 & Crepidium acuminatum (D.Don) Szlach. & Ái lan nhọn & Ter \\
\hline
\end{tabular}




\begin{tabular}{|c|c|c|c|}
\hline 58 & Crepidium calophyllum (Rchb.f.) Szlach. & Ái lan mỹ diệp & Ter \\
\hline 59 & Crepidium purpureum (Lindl.) Szlach. ${ }^{*}$ & Mai đất tím & Ter \\
\hline 60 & Cryptochilus cf. ctenostachyus Gagnep. & Ấn thiệt trâm & Epi \\
\hline 61 & Cryptochilus siamensis (Schltr.) Schuit. & Nỉ lan xiêm & Epi \\
\hline 62 & Cymbidium aloifolium (L.) Sw. & Đoản kiếm lô hội & Epi \\
\hline 63 & Cymbidium bicolor Lindl." & Đoản kiếm hai mầu & Ter \\
\hline 64 & Cymbidium erythrostylum Rolfe. & Bạc lan & Ter \\
\hline 65 & Cymbidium finlaysonianum Lindl. & Đoạn kiếm & Epi \\
\hline 66 & Dendrobium acerosum Lindl. & Hoàng thảo lá kim & Epi \\
\hline 67 & Dendrobium aduncum Wall. ex Lindl. & Hồng cầu & Epi \\
\hline 68 & Dendrobium aloifolium (B1.) Reichb.f. & Móng rồng & Epi \\
\hline 69 & Dendrobium anosmum Lindl. & Phi điệp & Epi \\
\hline 70 & Dendrobium bilobulatum Seidenf. & Phiếm đờn hai thùy & Epi \\
\hline 71 & Dendrobium blaoense Schuit. \& Peter B.Adams & Thạch mộc Việt Nam & Epi \\
\hline 72 & Dendrobium capillipes Rchb.f. & Kim điệp & Epi \\
\hline 73 & Dendrobium crepidatum Lindl. \& Paxt. & Ngọc vạn sáp & Epi \\
\hline 74 & Dendrobium cumulatum Lindl. ${ }^{*}$ & Hoàng thảo tích tụ & Epi \\
\hline 75 & Dendrobium delacourii Gouill. & Điểu lan & Epi \\
\hline 76 & Dendrobium densiflorum Lindl. & Thủy tiên mờ gà & Epi \\
\hline 77 & Dendrobium draconis Rchb.f. & Nhất điểm hồng & Epi \\
\hline 78 & Dendrobium ellipsophyllum T.Tang \& F.T.Wang ${ }^{*}$ & $\begin{array}{l}\text { Hoàng thảo hương } \\
\text { duyên }\end{array}$ & Epi \\
\hline 79 & Dendrobium exile Schltr. & Mộc lan đày & Epi \\
\hline 80 & Dendrobium farmeri Paxt. & Thủy tiên trắng & Epi \\
\hline 81 & Dendrobium gratiosissimum Reich.f. & $\begin{array}{l}\text { Lan ý thảo, Hoàng } \\
\text { thảo }\end{array}$ & Epi \\
\hline 82 & Dendrobium heterocarpum Wall. ex Lindl. & Nhất điểm hoàng & Epi \\
\hline 83 & Dendrobium indivisum (Blume) Miq. & $\begin{array}{l}\text { Hoàng thảo không } \\
\text { phân }\end{array}$ & Epi \\
\hline 84 & Dendrobium leonis (Lindl.) Reichb.f. & Sủ trầm & Epi \\
\hline 85 & Dendrobium lindleyi Steudel. & Vảy cá, Vảy rắn & Epi \\
\hline 86 & Dendrobium linguella Rchb.f. & Hương ve ni & Epi \\
\hline 87 & Dendrobium mannii Ridl. & Hoàng thảo xương khô & Epi \\
\hline 88 & Dendrobium metrium Kraenzl. & Hoàng thảo & Epi \\
\hline 89 & Dendrobium nathanielis Rchb.f. & Hoàng thảo móng rồng & Epi \\
\hline 90 & Dendrobium oligophyllum Gagn. & Hương duyên & Epi \\
\hline 91 & Dendrobium pachyglossum Par.\&. Reichb.f. & Mộc lan lưỡi dày & Epi \\
\hline
\end{tabular}




\begin{tabular}{|c|c|c|c|}
\hline 92 & Dendrobium palpebrae Lindl. & Trâm vàng & Epi \\
\hline 93 & Dendrobium parciflorum Rchb.f. ex Lindl. & Hoàng thảo hương lan & Epi \\
\hline 94 & Dendrobium polyanthum Wall. ex Lindl. & Thạch hộc vôi & Epi \\
\hline 95 & Dendrobium salaccense ( Bl.) Lindl. & Môc lan sa lắc & Epi \\
\hline 96 & Dendrobium secundum (Bl.) Lindl. & Báo hỉ & Epi \\
\hline 97 & Dendrobium simondii Gangnep. & Hoàng thảo Simond & Epi \\
\hline 98 & Dendrobium thyrsiflorum Reichb.f. & Thủy tiên mõ̃ gà & Epi \\
\hline 99 & Dendrobium truncatum Lindl. & Phong lan & Epi \\
\hline 100 & Dendrobium umbonatum Seidenf. & $\begin{array}{l}\text { Hoàng thảo môi hình } \\
\text { thuẫn }\end{array}$ & Epi \\
\hline 101 & Dendrobium uniflorum Griff." & Hoàng thảo nhất hoa & Epi \\
\hline 102 & Dendrolirium lasiopetalum (Willd.) S.C.Chen \& J.J.Wood & Nỉ lan lông & Epi, Ter \\
\hline 103 & Dienia ophrydis (J.Koenig) Seidenf. & Ái lan lá rộng & Ter \\
\hline 104 & Gastrochilus obliquus (Lindl.) Kuntze & Hàm lân tu & Epi \\
\hline 105 & Gastrochilus yunnanensis Schltr. & Hàm lân vân nam & Epi \\
\hline 106 & 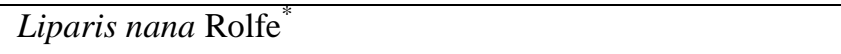 & Tai dê lùn & Ter \\
\hline 107 & Liparis viridiflora (Blume). Lindl. & Lan tai dê hoa xanh & Epi \\
\hline 108 & Luisia brachystachys (Lindl.) Bl. & Lụi chùm ngắn & Epi \\
\hline 109 & Luisia filiformis Hook.f." & Lan San hô sợi & Epi \\
\hline 110 & Luisia psyche Reichb.f. & Lụi mơ & Epi \\
\hline 111 & Luisia ramosii Ames ${ }^{*}$ & San hô & Epi \\
\hline 112 & Luisia sp. & Lụi & Epi \\
\hline 113 & Macropodanthus alatus (Holttum) Seidenf. \& Garay & Đại cước & Epi \\
\hline 114 & Malaxis sp. & Ailan & Ter \\
\hline 115 & Micropera pallida (Roxb.) Lindl. & Vi túi tái & Epi \\
\hline 116 & Micropera poilanei (Guillaumin) Garay ${ }^{*}$ & Vi túi Poilane & Epi \\
\hline 117 & Micropera thailandica (Seidenf. \& Smitinand) Garay & Vi túi Thái & Epi \\
\hline 118 & Microsaccus griffithii (E.C.Parish \& Rchb.f.) Seidenf. & Phong lan & Epi \\
\hline 119 & Mycaranthes pannea (Lindl.) S.C.Chen \& J.J.Wood ${ }^{*}$ & Nỉ len tã tơi & Epi \\
\hline 120 & Nervilia concolor (Blume) Schltr. & Trân châu xanh & Ter \\
\hline 121 & Nervilia fordii Averyanov" & Lan một lá & Ter \\
\hline 122 & Nervilia gracilis Averyanov" & Diệp tâm lan & Ter \\
\hline 123 & Nervilia plicata (Andrews) Schltr." & Trân châu xếp & Ter \\
\hline 124 & Nervilia simplex (Thouars) Schltr. & Trân châu & Ter \\
\hline 125 & Nervilia sp. & Trân châu & Ter \\
\hline 126 & Oberonia acaulis Griff. & Móng rùa không thân & Epi \\
\hline 127 & Oberonia caulescens Lindl. ${ }^{*}$ & Móng rùa có thân & Epi \\
\hline
\end{tabular}




\begin{tabular}{|c|c|c|c|}
\hline 128 & Oberonia gammiei King \& Pantl. & Móng rùa Gammi & Epi \\
\hline 129 & 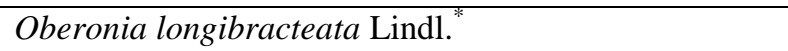 & Móng rùa lá bắc dài & Epi \\
\hline 130 & Oberonia lycopodioides (J.Koenig) Ormerod & Móng rùa hai đầu & Epi \\
\hline 131 & Oberonia nitida Seidenf. & Móng rùa đẹp & Epi \\
\hline 132 & Oberonia rufilabris Lindl. & Móng rùa môi đỏ & Epi \\
\hline 133 & Oberonia trochopetala Gagnep & Móng rùa & Epi \\
\hline 134 & Panisea albiflora (Ridl.) Seidenf. & Khúc thần hoa trắng & Epi \\
\hline 135 & Panisea uniflora Lindl. ${ }^{*}$ & Khúc thần một hoa & Epi \\
\hline 136 & Pelatantheria ctenoglossum Ridl. ${ }^{*}$ & $\begin{array}{l}\text { Bạt lan trâm, Lan môi } \\
\text { râu }\end{array}$ & Epi \\
\hline 137 & Phalaenopsis lobbii (Reichb.f) H.R.Sweet & Tục đoạn đốt & Epi \\
\hline 138 & Pholidota articulata Lindl. & Tục đoạn kế & Epi \\
\hline 139 & Pholidota imbricata Roxb. ex. Hook.f. & Tục đoạn kết hợp & Epi \\
\hline 140 & Pinalia acervata Kuntze ${ }^{*}$ & Nỉ lan trắng & Epi \\
\hline 141 & Pinalia cochinchinensis (Gagnep.) Schuit." & Nỉ lan Nam Bộ & Epi \\
\hline 142 & Pinalia dongnaiensis (Gagnep.) S.C.Chen \& J.J.Wood ${ }^{*}$ & Nỉ lan Đồng Nai & Epi \\
\hline 143 & Pinalia floribunda Kuntze & Lan len nhiều hoa & Epi \\
\hline 144 & Polystachya concreta (Jacq.) Garay \& H.R. Sweet & Đa phướng & Epi \\
\hline 145 & Pomatocalpa spicatum Breda, Kuhl \& Hasselt & Phong lan & Epi \\
\hline 146 & Pteroceras compressum (Blume) Holttum ${ }^{*}$ & Môi sừng & Epi \\
\hline 147 & Pteroceras teres (Blume) Holttum & Dực giác tròn & Epi \\
\hline 148 & Rhynchostylis coelestis Reichb.f. & Cờ lao, Ngọc điểm & Epi \\
\hline 149 & Rhynchostylis gigantea (Lindl.) Ridl. & $\begin{array}{l}\text { Lan lưỡi bò, Ngọc } \\
\text { điểm }\end{array}$ & Epi \\
\hline 150 & Rhynchostylis retusa (L.) Blume ${ }^{*}$ & Ngọc điểm đuôi cáo & Epi \\
\hline 151 & Robiquetia succisa (Lindl.) Seidenf. \& Garay ${ }^{*}$ & Túi chùy thắt & Epi \\
\hline 152 & Smitinandia helferi (Hook.f.) Garay & Lan Mã lai vành & Epi \\
\hline 153 & Smitinandia micrantha (Lindl.) Holttum ${ }^{*}$ & Lan Smitinan hoa nhỏ & Epi \\
\hline 154 & Staurochilus fasciatus (Reichb. f.) Ridl. & Hổ bì & Epi \\
\hline 155 & Taeniophyllum daroussinii Tixier \& Guillaumin ${ }^{*}$ & Căn diệp xuân lộc & Epi \\
\hline 156 & Taeniophyllum pusillum (Willd.) Seidenf. \& Ormerod & Đai điệp tà & Epi \\
\hline 157 & Thecostele alata (Rchb.f.) Par.\& Rchb.f. ${ }^{*}$ & Củ chén & Epi \\
\hline 158 & Thelasis micrantha (Brongn.) J.J.Sm. & Lan củ dẹt nhọn & Epi \\
\hline 159 & Thelasis pygmaea (Griff.) Lindl. & Lan củ lùn dẹt & Epi \\
\hline 160 & Thrixspermum centipeda Lour. & $\begin{array}{l}\text { Lan xương cá, Mao tử } \\
\text { rít }\end{array}$ & Epi \\
\hline 161 & Thrixspermum hystrix (B1.) Reichb.f. & Mao tử nhiếm & Epi \\
\hline
\end{tabular}




\begin{tabular}{|c|l|l|c|}
\hline 162 & Thrixspermum leucarachne Ridl. & Mao tử & Epi \\
\hline 163 & Thrixspermum sp. & Mao tử Nam Cát Tiên & Epi \\
\hline 164 & Thrixspermum trichoglottis (Hook.f.) Kuntze & Mao tử lưỡi & Epi \\
\hline 165 & Thunia alba (Lindl.) Rchb.f. & Hạc đỉnh trắng & Ter \\
\hline 166 & Thunia pulchra Rchb.f. & Bạch hạc nhỏ & Ter \\
\hline 167 & Trichoglottis retusa Blume & Mao thiệt tà & Epi \\
\hline 168 & Trichoglottis seidenfadenii Aver. & Mao thiệt Seidenfaden & Epi \\
\hline 169 & Trichotosia pulvinata (Lindl) Kraenzl. & Mao lan gối & Epi \\
\hline 170 & Trichotosia velutina (Lodd. ex Lindl.) Kraenzl. & Mao lan lông & Epi \\
\hline 171 & Tropidia curculigoides Lindl & Lan đất bông ngắn & Ter \\
\hline 172 & Vanda denisoniana Benson \& Rchb.f. & Mỹ dung dạ hương & Epi \\
\hline 173 & Vanilla aphylla Blume & Vani không lá & Epi \\
\hline
\end{tabular}

Notes: Epi: Orchid, Lit: Stone orchid, Ter: Cymbidium, Sap: saprophytic orchid; * Additional species for the South East.

The results in Table 1, species composition of the orchid family(Orchidaceae) in South East region is quite diverse and rich with 173 species, accounting for $14.41 \%$ of the total number of orchid species available in Viet Nam (1.200 species) (Averyanov, et al., 2003; Averyanovand et al., 2017a; Pham Hoang Ho, 2003). The most diverse is the genus (Dendrobium) with 36 species, accounting for $20.80 \%$ of the total species of the orchid family currently in the study area; followed is genus (Bulbophyllum) with 16 species, accounting for 9.25\%; genus Coelogyne and Oberonia have 8 species, accounting for $4.62 \%$, genus Calanthe has 7 species accounting for $4.05 \%$, genus Cleisostoma and Nervilia have 6 species accounting for 3.47\%, genus Luisia, Aerides, Thrixspermum have 5 species accounting for $2.9 \%$. The remaining genera only 1 to 4 species, accounting for low rate (Averyanov et al., 2013, Averyanovand et al., 2017a; Nguyen Thien Tich, 2001; Tran Hop, 1998).

\subsection{Diverse in life form}

The life forms of species of the Orchid family (Orchidaceae) in South East region is very diverse and distributed in many different habitats, one species can have one or two different life forms. Table 1 shows that the orchid life form group (Epi) has the most number of species with 144 species, accounting for $83.23 \%$ of the total number of species in the study area, this group often cling to large trees in the forests, evergreen moist; next is cymbidium (Ter) group with 26 species, accounting for $15.03 \%$, this group often grows on soil with moist rotting layer or meat soil under the buried forest; Saprophytic orchid group (Sap) has 1 species, accounting for $0.58 \%$; Stone orchid group (Lit) has 2 species, accounting for $1.15 \%$.

\subsection{Diversity of rare genetic resources}


The identification of endangered orchid species plays an extremely important role in the conservation of genetic resources and sustainable development of this particularly valuable resource. Table 1 shows that, there are 6 species of orchids (accounting for $3,46 \%$ of total species) in the South East region of conservation value according to the Vietnam Red Data Book, (2019). In which, there are 3 species of orchid ranked endangered, the level (EN) is Agrostophyllum planicaule, Dendrobium heterocarpum, Pinalia dongnaiensis and 3 species ranked will be endangered, the level (VU) is Dendrobium draconis, Dendrobium farmeri, Coelogyne trinervis, these species are narrowly distributed. But often affected by their habitat and always exploited for commercial purposes (Ministry of Science and Technology, 2019).

\subsection{Diversity in value use}

All species in the orchid family (Orchidaceae) in the Southern East region are of economic and commercial value, used as an ornamental because they are easy to grow and care for, they often give beautiful color and fragrance. The results of the study have also identified 13 species (accounting for $7.51 \%$ of the total species) of medicinal value including: Acriopsis liliifolia, Aerides falcata, Appendicula cornuta, Calanthe triplicata, Cymbidium aloifolium, Dendrobium aduncum, Dendrobium gratiosissimum, Nervilia fordii, Pholidota articulata, Pholidota imbricata, Rhynchostylis retusa, Tropidia curculigoides, Anoectochilus roxburghii (Do Tat Loi, 2009; Nguyen Thien Tich, 2001; Pham Hoang Ho, 2006; Vo Van Chi, 2012).

\section{Conclusion}

The study results have identified the orchid family (Orchidaceae) in the South East with 173 species belonging to 50 genera. In which, all species have ornamental value, 13 species have medicinal value, 6 species in the Viet Nam Red Data Book, (2019). The life forms of species of the orchid family (Orchidaceae) is divided into 4 groups: Orchids (Epi) has 144 species, Cymbidium (Ter) has 26 species, saprophytic orchid (Sap) has 1 species, Stone orchid (Lit), there were 2 species. Added 60 species to forest orchid flora of Southern East Viet Nam.

\section{Reference}

Do Tat Loi (2009). Vietnamese medicinal plants and herbs. Hanoi: Medical Publishing House.

F. Gagnepain \& A. Guillaumin "Orchidaceae \& Apostasiaceae", Flore Générale de l'Indochine, Vol 6, 1932-1934, pp.142-647.

K.Karthigeyan, J.Jayanthi, R.Sumathi \& J.S.Jalal (2004). A review of the orchid diversity ofAndaman \& Nicobar Islands, India, Richardia, 9-85. 
L. Averyanov, P.J. Cribb, K.L. Phan \& T.H. Nguyen (2003). Slipper Orchids of Vietnam: with an Introduction to the Flora of Vietnam. Kew: Royal Botanic Gardens, 308p.

L.V. Averyanov (2013). The Orchids Of Vietnam Illustrated Survey, Part 4. Subfamily Epidendroideae, Turczaninowia, pp.5-163.

L.V. Averyanov, A.L. Averyanova (2003). Updated Checklist of the Orchids of Vietnam, Vietnam National University Publishing House, 101 p.

L.V. Averyanovand T.V. Maisak (2017a). New data on orchid diversity of Vietnam, 2012-2016. P. 77-80. Taxonomy and evolutionary morphology of plants. Materials of the Conference dedicated to 85 anniversary of V.N. Tikhomirov. January 31-3 February, 2017. Moscow, MAKS Press. 496 pp.

Ministry of Science and Technology (2007). Vietnam Red Book - Botanical Section. Hanoi: Science and Technology Publishing House, pp. 399-478.

Nguyen Thien Tich (2001). Lan Vietnam, volume 1, Agriculture Publishing House, Ho Chi Minh City, 424 pages.

P. Cribb (1998). The Genus Paphiopedilu, Royal Botanic Gardens Kew, 427 p.

Pham Hoang Ho (2003). Orchidaceae - Vietnamese Orchids, Book 3. HCMc: Young Publishing House, pp. 760-968.

Pham Hoang Ho (2006). A medicinal plant in Vietnam. HCMc: Youth Publishing House, pages 649-654.

Sterling, E.J., Hurley, M.M. and L.D.Minh (2007). Lịch sử tự nhiên của Việt Nam. Yale University Press New Haven and London, trang 277-333.

Tran Hop (1998). Vietnamese Orchid. HCMc: Agriculture Publishing House.

Vo Van Chi (2012). Dictionary of Vietnamese medicinal plants, (volume 1, 2). Hanoi: Medical Publishing House. 